\title{
RESEÑAS \\ La justicia de los derechos humanos: Una justicia singular que no debe darse por sentada
}

\author{
Rita Lages (iD) \\ Universidad de Chile
}

g Laurence Burgorgue-Larsen, Les 3 cours régionales des droits de l'homme in context: La justice qui nallait pas de soi, Paris, Éditions A. Pedone, 2020, 588 páginas.

En septiembre de 2020 fue publicada la más reciente obra monográfica de la profesora Burgorgue-Larsen, Les 3 cours régionales des droits de l'homme in context: La justice qui nallait pas de soi, dedicada al «estudio de los tres únicos mecanismos jurisdiccionales eficaces que funcionan en Europa, América y África, y que han sido creados para proteger los derechos humanos» (17). Pocos meses después, el 17 de diciembre, se publicó el primer Reporte conjunto sobre su jurisprudencia 2019 (Corte IDH y otros, 2020). Esta publicación consiste en un anuario de jurisprudencia comentada de las sentencias más importantes dictadas en cada período por el Tribunal Europeo de Derechos (TEDH), la Corte Interamericana de Derechos Humanos (Corte IDH) y la Corte Africana de Derechos Humanas, y corresponde a un compromiso asumido en la primera reunión del Foro Permanente de Diálogo Institucional realizado en 2019. ${ }^{1}$ Este foro fue establecido con ocasión del cuadragésimo aniversario de la entrada en vigor de la Convención Americana sobre Derechos Humanos (CADH) y de la creación de la Corte IDH, por los presidentes de las cortes africana, europea e interamericana de derechos humanos y con el propósito de «fortalecer la protección de los derechos humanos y el acceso a la justicia internacional de las personas bajo jurisdicción de los tres tribunales». ${ }^{2}$

1. La primera reunión del Foro Internacional de Derechos Humanos se realizó el 28 y 29 de octubre de 2019 en Kampala, capital de Uganda.

2. Declaración Conjunta de los presidentes de la Corte Africana de Derechos Humanos y de los Pueblos, del Tribunal Europeo de Derechos Humanos y de la Corte Interamericana de Derechos Humanos, en ocasión del cuadragésimo aniversario de la entrada en vigor de la Convención Americana sobre Derechos Humanos y de la creación de la Corte Interamericana de Derechos Humanos (Declaración de San José), de 18 de julio de 2018. 
A nuestro parecer, esta feliz coincidencia temporal constituye un ejemplo representativo de la actualidad e importancia de la obra ahora reseñada. Conocer el origen, funcionamiento y competencias de los tribunales internacionales, en particular de las tres cortes regionales de derechos humanos, es fundamental para entender el proceso de «globalización judicial» (Slaughter, 2000) en el que nos encontramos, caracterizado por una creciente interacción judicial y un activo diálogo jurisprudencial mediante relaciones «verticales» y sobre todo «horizontales» entre los tribunales. En el ámbito de los derechos humanos, la globalización judicial ha dado lugar a una protección multinivel de los derechos fundamentales en virtud de la coexistencia de jurisdicciones supra, trans y nacionales que interactúan y se influencian recíprocamente mediante un proceso de cross-fertilization (Slaughter, 2003) que contribuye a la armonización jurisprudencial y a la interpretación uniforme de los estándares internacionales en materia de derechos humanos. El diálogo jurisprudencial ha adoptado la forma de mecanismos institucionalizados (por ejemplo, la cuestión prejudicial del artículo 267 TFUE en el ámbito del derecho de la Unión Europea), y más frecuentemente, de mecanismos informales (Martins y Roque, 2014: 37-40). ${ }^{3}$ Entre estos últimos se insertan justamente el mencionado Foro Permanente de Diálogo Institucional y el Reporte Conjunto de Jurisprudencia 2019.

Les 3 cours es un libro ambicioso que tiene por objetivo, en palabras de la autora, «descifrar la justicia de los derechos humanos, a través del tiempo y el espacio; la de los tribunales y no la de los comités; los tribunales en funcionamiento» (16). Sus 588 páginas se estructuran en seis capítulos esparcidos por tres títulos, además de un capítulo preliminar, un prólogo, un epílogo y una exhaustiva bibliografía. Metodológicamente, el libro adopta un interesante enfoque multidisciplinario en el que el análisis jurídico comparativo se enriquece con elementos de historia, política y sociología jurídica.

Desde el capítulo introductorio («Création»), dedicado a analizar los factores geopolíticos (23-45) y diplomáticos (45-72) que han originado la creación de cada

3. Utilizando la sistematización realizada por Martins y Roque (2014: 39-40), la interacción jurisprudencial de tipo dialógico utilizada por los tribunales ha asumido la forma de: a) diálogo directo mediante redes judiciales que, entre sus funciones y actividades, se encargan de intercambiar información, organizar seminarios, publicar informes u obras colectivas sobre derechos humanos y fundamentales; b) diálogo indirecto, mediante la consulta de sentencias de otras jurisdicciones para saber cómo aquellas han resuelto problemas o cuestiones jurídicas similares y la citación mutua; y c) diálogo a través de la recepción de doctrina en sus fallos, participando de este modo en el debate académico y doctrinal respecto de un tema en particular. Burgorgue-Larsen (2020: 426 y ss.) utiliza el término diplomacia judicial y académica para referirse a las reuniones y encuentros entre los jueces de las tres cortes con sus pares nacionales e internacionales para compartir intereses y preocupaciones comunes o su participación como conferencistas, docentes invitados de actividades académicas universitarias. Véase, también, Slaughter (2003: 195 y ss.). 
uno de estos sistemas regionales de protección, y mediante su completísimo estudio, la profesora Burgorgue-Larsen resalta la idea, reafirmada en cada uno de los tres títulos que se le siguen — «Évolution», «Interprétation» y «Application»-, de que la constitución y desarrollo de un sistema internacional (y regionales) de tutela de los derechos humanos es tributario de un proceso complejo, largo y paulatino de construcción político-jurídica de un consenso ético mínimo común de la humanidad respecto de la persona humana, cuya existencia se debe a la confluencia de ciertos acontecimientos históricamente irrepetibles, a la determinación de ciertos personajes y a la acción de determinadas instituciones en los tres continentes; pero cuya evolución ha estado marcada por desconfianzas iniciales de algunos Estados, en el caso europeo, y posiciones refractarias hasta el presente, en los casos americano y africano, a la judicialización de los sistemas regionales de protección y competencia obligatoria de las respectivas cortes que, pese a sus diferencias, cuentan con una actuación limitada en cuanto a la definición de su estructura y a la elección de los derechos consagrados en cada uno de los catálogos. Esto remite directamente a dos de las más complejas cuestiones sobre la actual justicia internacional de derechos humanos: su efectividad y legitimidad. Estos dos temas son magistralmente tratados en los capítulos primero («L’efficacité difficile») y segundo («La légitimité aléatoire»), respectivamente.

Los capítulos tercero («L' existence du décloisonnement») y cuarto («Les effets du décloisonnement») del título segundo versan profusamente sobre la interpretación de los tratados de derechos humanos realizados por las tres cortes. En ellos, la autora da cuenta de las similitudes y diferencias en cuanto a los principios y reglas de interpretación. Así, por ejemplo, mientras el principio de no regresión encuentra en las convenciones europea e interamericana una base jurídica sólida (artículos 53 de $\mathrm{CEDH}$ y 29 de la $\mathrm{CADH}$ ) que impone al juez una obligación negativa (no realizar una interpretación de las normas de derechos humanas que menoscabe o disminuya los umbrales y estándares de protección nacionales y/o internacionales ya adquiridos) y otra positiva (de progresividad mediante la adopción de interpretaciones que den la mejor protección posible, ya que se trataría más bien de una cláusula de «trato más favorable»); en el sistema africano, la solución elegida fue habilitar el juez para aplicar, además de las normas de la Carta Africana, disposiciones contenidas en otros tratados internacionales, externos al sistema africano, que sean pertinentes en materia de derechos humanos y se encuentren vigentes en el Estado parte (artículo 7 del Protocolo de Ouagadougou). No obstante las diferencias técnicas en los textos jurídicos convencionales mencionados, en las tres cortes hay una práctica de lo que la autora designa como décloisonnement (o «descompartimentación»), es decir, de apertura a las fuentes externas (247), independientemente de su naturaleza (convencional, jurisprudencial u otra) o de su origen (ya sean procedentes de jurisdicciones universales, especializadas, regionales o nacionales). En los sistemas europeo y americano, esto significó la adopción de una «descompartimentación rebelde» mediante 
el recurso a la interpretación evolutiva de los instrumentos regionales e internacionales de protección, combinada con, en el caso europeo, la interpretación teleológica (249-250); o, en el caso de la CADH, con una interpretación pro persona, apoyada en el reconocimiento expreso de la «cláusula de no regresividad» en la Convención (artículo 29 de la CADH). En el sistema africano, se adoptó una "descompartimentación fiel» a lo establecido en las cláusulas de apertura de los artículos 60 y 61 de la Carta Africana y 7 del Protocolo, y especialmente, a la voluntad de los Estados. Para la autora, el décloisonnement no está exento de paradojas en cuanto a la utilización de las bases jurídicas para la elección de fuentes externas por parte del tribunal africano, y de singularidades vinculadas a la relación entre las tres cortes y las respectivas jurisdicciones nacionales (286-287).

La descompartimentación (entendida, recuérdese, como la apertura de las cortes a las fuentes externas) se ha traducido en un continuo diálogo judicial internacional cuyos efectos son analizados en el capítulo cuarto. Para la académica francesa, antigua magistrada y presidenta del Tribunal Constitucional de Andorra, esos efectos son dos. El primero, el incremento de la protección convencional («Laccroissement de la protection») resultante, por un lado, de la modificación de los textos de protección por vía de la descompartimentación interpretativa respecto de la definición del contenido y alcance de los derechos existentes y el enriquecimiento del primero, $y$, por otro, de la ampliación de las obligaciones estatales, especialmente en los ámbitos de la no discriminación y de la vulnerabilidad. El segundo efecto, el aumento de los cuestionamientos («Laccroissement des contestations») por parte de Estados, organizaciones conservadoras de la sociedad civil o incluso de jueces al interior de las cortes a través de la emisión de opiniones separadas que revelan fracturas sobre las técnicas generales de interpretación («décloisonnement contesté», en el caso del TEDH y de la Corte IDH) o divergencias de carácter técnico («décloisonnement discuté», en el caso de la Corte Africana).

En el tercer título del libro, relativo a la aplicación del derecho, la autora aclara que adopta una perspectiva comprensiva del concepto. Esta perspectiva comprensiva implica entender la aplicación del derecho como la operación de dar efecto a una regla jurídica, cualquiera que sea su origen o régimen práctico (363, notas 1 y 2). Así definido el término adaptación utilizado, la autora examina, por un lado, los mecanismos relativos a la aplicación de los instrumentos convencionales adoptados en los tres sistemas regionales y, por otro, los mecanismos que permiten la ejecución de las decisiones judiciales supranacionales dictadas por las tres cortes (363). En este, como en los otros temas analizados en los capítulos precedentes, se identifican posiciones dualistas que, según la autora, por ser extremas tienden a simplificar la complejidad propia de la realidad. En este caso, de un lado, la perspectiva institucionalista y, por el otro, la perspectiva realista. Los institucionalistas focalizan el análisis de la aplicación del derecho internacional en los positivos de la pertenencia de un Estado a una or- 
ganización internacional (entre otros, la búsqueda de consensos y soluciones armoniosas, la cooperación como método para lograr objetivos comunes), mientras que para los realistas su punto de partida es la convicción de que los Estados se mueven exclusivamente por sus intereses nacionales y solo existe la legitimidad y la fuerza de aquellos en las relaciones internacionales.

La tesis sostenida en este título tercero es la de que la aplicación efectiva de las normas y principios de los convenios regionales de derechos humanos y la ejecución de las decisiones judiciales solo son posibles si concurren «sinergias»: como elucida la autora, acciones coordinadas ( 365 , nota 7 ), internacionales, regionales y regionales de múltiples actores, organismos o instituciones (estatales y no estatales), con el propósito de: a) alentar o presionar los Estados para cumplir sus responsabilidades internacionales en la materia (capítulo 5, «Les synergies de l'incitation»), o b) controlar y coordinar la actuación del Estado (capítulo 6, «Les synergies du control»). Las «sinergias de incitación» son de dos tipos y, tal como advierte la profesora Burgorgue-Larsen, para que puedan cumplir su propósito deben complementarse: la «incitación constitucional» (368-390) y la «incitación dialógica»(390-438). La primera está relacionada con el ya conocido fenómeno de la «constitucionalización del derecho internacional»o la «internalización del derecho constitucional», que se expresa en la apertura constitucional al derecho internacional de los derechos humanos posibilitada por cláusulas "pasarelas» presentes en muchas de las cartas fundamentales de los varios Estados parte de los sistemas regionales de protección. La segunda contribuye también y significativamente a la «humanización» del ordenamiento jurídico interno como consecuencia del reconocimiento de la importancia de la función interpretativa del derecho internacional de los derechos humanos para la aplicación de los estándares internacionales elaborados por las tres cortes regionales a los ordenamientos jurídicos internos, ya sea por vía de un «diálogo judicial» (391-408), mediante un control de convencionalidad, mayormente desarrollado por la jurisprudencia interamericana, o por un «diálogo político» (409-438) realizado por las ONG y las instituciones nacionales de derechos humanos («diplomacia cívica») o por jueces, cuando realizan encuentros con otros jueces internacionales y nacionales para compartir experiencias, intereses o preocupaciones comunes en este ámbito, o cuando participan de actividades académicas en colaboración con instituciones universitarias («diplomacia judicial y académica»). Las «sinergias de control» remiten a la ejecución de las decisiones judiciales y formas de reparación establecidas en los tres sistemas regionales de protección.

También en esta materia la profesora Burgorgue-Larsen comparte minuciosamente su comprensión acerca de los elementos comunes y disímiles a los tres sistemas de protección. En los tres existen mecanismos de control regional (442 y ss.) y de coordinación nacional (466 y ss.). En los sistemas europeo y africano se les asignó a los respectivos órganos intergubernamentales del Consejo de Europa (el Comité 
de Ministros) y de la Unión Africana (Consejo Ejecutivo) la función de controlar la ejecución del cumplimiento de las sentencias y sus medidas de reparación; en el sistema interamericano esa competencia no fue explícitamente atribuida a la Asamblea General, en tanto órgano intergubernamental de la Organización de Estados Americanos. En el sistema europeo hay una «sinergia global» (444) o «tridimensional» (445), pues participan del procedimiento de supervisión del cumplimiento de las obligaciones derivadas de las sentencias condenatorias del TEDH (obligaciones de cesar el ilícito, evitar su repetición y reparar el daño sufrido) el Comité de Ministros, la Asamblea Parlamentaria a través de informes y el propio TEDH mediante el procedimiento de las «sentencias piloto» en las que dicho tribunal asiste y orienta a los órganos políticos sobre las concretas medidas que los Estados deben adoptar para asegurarse una correcta ejecución de sus sentencias. En el sistema interamericano, esa sinergia es «parcial» (452), pues la decisión de los Estados de no atribuir a ningún órgano político de la OEA la función de vigilancia, la Comisión y Corte IDH se han involucrado en el seguimiento de la aplicación de las recomendaciones formuladas por la primera y de la ejecución de las sentencias dictadas por la segunda. Por el contrario, el sistema africano, el más joven de los tres, se caracteriza por la ausencia de un enfoque sinérgico (458). Complementariamente, se han desarrollado mecanismos de coordinación nacional (466) de carácter gubernamental y legislativo.

El libro termina tal como empezó, o sea, con un acápite de reflexiones en un tono más personal, así nos parece, pero no menos riguroso, acerca de la justicia de los derechos humanos. Si en las reflexiones iniciales («Singulière justice») la autora nos retrotrae al momento de la creación de los tres sistemas, que no ha estado exenta de dificultades, para decirnos que el contexto político de cada continente - marcado por las vivencias de ciertos eventos históricos (la Segunda Guerra Mundial y la expansión del comunismo; los regímenes presidenciales autoritarios en América o la colonización de África) - ha impulsado la emergencia de una justicia creada por los Estados para limitar su poder soberano en nombre de la dignidad de los seres humanos, a quienes se les reconocen derechos que los Estados deben respetar y garantizar. En las reflexiones finales («Entre le passé et le présent, quel futur?»), con la mirada en el porvenir, nos señala que ante los actuales y crecientes cuestionamientos, magnificados por discursos políticos populistas y autoritarios, a esta «justicia singular», se requiere una «vigilancia indispensable» y una «resistencia necesaria» (494) para preservar el acervo normativo y jurisprudencial que tanto costó alcanzar. El mensaje central del libro nos parece claro y particularmente relevante en nuestra «modernidad líquida» en la que, como apuntó Zygmunt Bauman, la incertidumbre es una constante: el reconocimiento universal y la tutela jurisdiccional de los derechos humanos no deben darse por sentados.

En cuanto a la bibliografía final, nos parece digno de mención su notable extensión, la diversidad temática, autoral e idiomática, y la coherente y cuidada ordenación 
según temas (creación, interpretación y aplicación), fecha de creación de cada sistema (Europa, América y África), la naturaleza de la fuente bibliográfica (monografías y artículos) y metodología utilizada (obras que abordan los sistemas regionales de protección desde una perspectiva comparada).

En resumen, encontramos en Les 3 cours todas las características de una gran obra de la literatura jurídica que, sin duda, tendrá seguramente un lugar obligatorio y privilegiado en las bibliotecas de las universidades y otras instituciones, y de todas aquellas personas interesadas y estudiosas de la temática de los derechos humanos y de su protección jurídica.

\section{Referencias}

Corte Interamericana de Derechos Humanos, Corte Europea de Derechos Humanos y Corte Africana de Derechos Humanos y de los Pueblos (2020). Joint Law Report 2019: African Court on Human and Peoples' Rights, European Court of Human Rights and Inter-American Court of Human Rights. San José de Costa Rica. Disponible en https://bit.ly/3iNS97d.

Martins, Ana Maria Guerra y Miguel Prata Roque (2014). «A tutela multinível dos direitos fundamentais. A posição do Tribunal Constitucional Português». Ponencia presentada en la Conferência Trilateral dos Tribunais Constitucionais Espanhol, Italiano e Português realizada el 16 a 18 de octubre de 2014, Santiago de Compostela. Disponible en https://bit.ly/370LFw5.

SLAUghter, Anne-Marie (2000). «Judicial globalization». Virginia Journal of International Law, 40 (4): 1.103-1.124.

-. (2003). «Global community of courts». Harvard International Law Journal, 44 (1): 191-220.

\section{Sobre la autora}

Rita Lages es licenciada en Derecho por la Universidad de Coimbra, Portugal. Máster en Ciencias Jurídico-Comunitarias (LL.M.) por la Universidad de Coimbra, Portugal. Profesora asistente del Departamento de Derecho Internacional de la Universidad de Chile e investigadora del Centro de Derechos Humanos. Su correo es rlages@ derecho.uchile.cl. (D) https://orcid.org/oooo-0001-6260-2531. 
El Anuario de Derechos Humanos es una publicación semestral de referencia y consulta en materia de derechos humanos y campos afines. Busca ser un espacio de discusión de los temas centrales en el ámbito nacional e internacional sobre derechos humanos. Es publicado desde 2005 por el Centro de Derechos Humanos de la Facultad de Derecho de la Universidad de Chile.

\author{
EDITORA \\ Claudia Iriarte Rivas \\ ciriarter@derecho.uchile.cl \\ SITIO WEB \\ anuariocdh.uchile.cl \\ CORREO ELECTRÓNICO \\ anuario-cdh@derecho.uchile.cl \\ LICENCIA DE ESTE ARTÍCULO \\ Creative Commons Atribución Compartir Igual 4.o Internacional
}

\author{
\% \\ La edición de textos, el diseño editorial \\ y la conversión a formatos electrónicos de este artículo \\ estuvieron a cargo de Tipográfica \\ (www.tipografica.io)
}

